\title{
Faraday Instability in a Surface-Frozen Liquid
}

\author{
P. Huber, ${ }^{1, *}$ V.P. Soprunyuk, ${ }^{1}$ J.P. Embs, ${ }^{1}$ C. Wagner, ${ }^{2}$ M. Deutsch,${ }^{3}$ and S. Kumar ${ }^{4}$ \\ ${ }^{1}$ Technische Physik, Universität des Saarlandes, 66041 Saarbrücken, Germany \\ ${ }^{2}$ Experimentalphysik, Universität des Saarlandes, 66041 Saarbrücken, Germany \\ ${ }^{3}$ Physics Department, Bar-Ilan University, Ramat-Gan 52900, Israel \\ ${ }^{4}$ Department of Chemical Engineering and Materials Science, \\ University of Minnesota, Minneapolis, MN 55455, U.S.A.
}

(Dated: July 24, 2013)

\begin{abstract}
Faraday surface instability measurements of the critical acceleration, $a_{\mathrm{c}}$, and wavenumber, $k_{\mathrm{c}}$, for standing surface waves on a tetracosanol $\left(\mathrm{C}_{24} \mathrm{H}_{50}\right)$ melt exhibit abrupt changes at $T_{s}=54{ }^{\circ} \mathrm{C}$, $\sim 4{ }^{\circ} \mathrm{C}$ above the bulk freezing temperature. The measured variations of $a_{\mathrm{c}}$ and $k_{\mathrm{c}}$ vs. temperature and driving frequency are accounted for quantitatively by a hydrodynamic model, revealing a change from a free-slip surface flow, generic for a free liquid surface $\left(T>T_{s}\right)$, to a surface-pinned, no-slip flow, characteristic of a flow near a wetted solid wall $\left(T<T_{s}\right)$. The change at $T_{s}$ is traced to the onset of surface freezing, where the steep velocity gradient in the surface-pinned flow significantly increases the viscous dissipation near the surface.
\end{abstract}

PACS numbers: 68.10.-m, 47.20.Ma, 61.25.Em, 64.70.Dv

Spatial confinement of a liquid often changes its properties markedly. For example, superheating above the equilibrium melting temperature [1] and order quenching upon freezing [2] were observed under confinement only. In particular, flow under confinement is important for processes ranging from tribology to protein folding to transport through ion channels in cell membranes 3]. A transition from a liquid-like to a granular-solid-like shear response was observed at nano-scale confinements [4]. The surface freezing (SF) effect [5], where a solid monolayer forms at the surface of a pure normal-alkane $\left(\mathrm{C}_{n} \mathrm{H}_{2 n+2}\right)$ melt, provides a unique system for studying semi-confined flow at a solid-liquid interface. The abrupt onset of SF at $T_{s}$ allows one to switch on (and off) the solid phase by a small temperature variation. Understanding such interfaces would also elucidate the role of flow in nucleation and growth processes of crystals from melts, which are dominated by such interfaces [6].

SF occurs in melts of several chain molecules (alkenes, alcohols, semi-fluorinated alkanes, diols, $\mathrm{C}_{i} \mathrm{E}_{j}$ ) [] , and at both liquid/liquid [8] and liquid/solid [9] interfaces. Related surface ordering effects were observed in melts of polymers comprising alkyl chains in the backbone or as side chains 10], in liquid alloys [1], and in several liquid crystals [12]. While the structural and thermodynamic aspects of SF have been studied in great detail [13], the influence of surface ordering on macroscopic near-surface flows has received little attention to date in this sizable, technologically-important class of materials.

To study this issue, we employ the Faraday instability, which forms standing wave patterns (SWP) at the free surface of a vertically-vibrated liquid [14]. By virtue of its simplicity, this instability is outstanding among pattern-forming systems and a detailed theoretical description has been achieved. The SWP formed depend sensitively on, and allow a detailed study of, the changes in the surface hydrodynamics upon SF. As this study of SF demonstrates, the instability can therefore be employed to explore physical processes which are difficult to access by other, classical means. It also exemplifies the more general class of parametric instabilities, which are of interest in a broad range of areas including granular media, plasmas, nonlinear optics, reaction-diffusion systems, and condensed-matter physics 15 .

The sample used is n-tetracosane, $\mathrm{C}_{24} \mathrm{H}_{50}$. With $T_{s}=$ $54{ }^{\circ} \mathrm{C}$ and a bulk freezing temperature $T_{b}=50{ }^{\circ} \mathrm{C}$, it exhibits the largest temperature range of $\mathrm{SF}$ of all pure n-alkanes [5]. Our circular, 18-cm diameter, sample cell was filled with $300 \mathrm{~g}$ of $\mathrm{C}_{24} \mathrm{H}_{50}$ (Aldrich, $99.9 \%$ pure) to a height of $h=1.5 \mathrm{~cm}$ above the cell bottom. The cell is immersed in a thermostatted water bath yielding good temperature stability $\left( \pm 0.05{ }^{\circ} \mathrm{C}\right)$ and minimizing temperature gradients between the surface and bulk. The bath is vibrated sinusoidally in the vertical direction at a driving frequency $f$ by an electromechanical shaker. The SWP are tracked and recorded using a CCD camera and stroboscopic illumination [16].

Two characteristic quantities were measured: the critical acceleration, $a_{\mathrm{c}}$, required for destabilizing the flat surface to form the SWP, and the critical wavenumber, $k_{\mathrm{c}}$, which defines the spatial periodicity of the SWP at the onset of the instability. Square SWP (inset of Fig. 1(a)) were found for all $T$ and $f$ investigated. Fourier transforms were used to determine $k_{\mathrm{c}}$ from each pattern. The $T$-dependence of $k_{\mathrm{c}}$ and $a_{\mathrm{c}}$ at fixed $f=150 \mathrm{~Hz}$ is shown in Fig. 11 Upon cooling from $58{ }^{\circ} \mathrm{C}$ to $54{ }^{\circ} \mathrm{C}, k_{\mathrm{c}}$ remains roughly constant. At $54^{\circ} \mathrm{C}$, however, a $\sim 60 \mathrm{~m}^{-1}$ jump is observed in $k_{\mathrm{c}}$. Below $T=54{ }^{\circ} \mathrm{C}, k_{\mathrm{c}}$ increases linearly with decreasing $T$. No cooling/heating hysteresis is observed. Similar behavior is seen for $a_{\mathrm{c}}$ : it is roughly constant for $54^{\circ} \mathrm{C}<T<58^{\circ} \mathrm{C}$, at $54^{\circ} \mathrm{C}$ it jumps by $\sim 20 \%$, and it is roughly constant for $T<54^{\circ} \mathrm{C}$. As the 

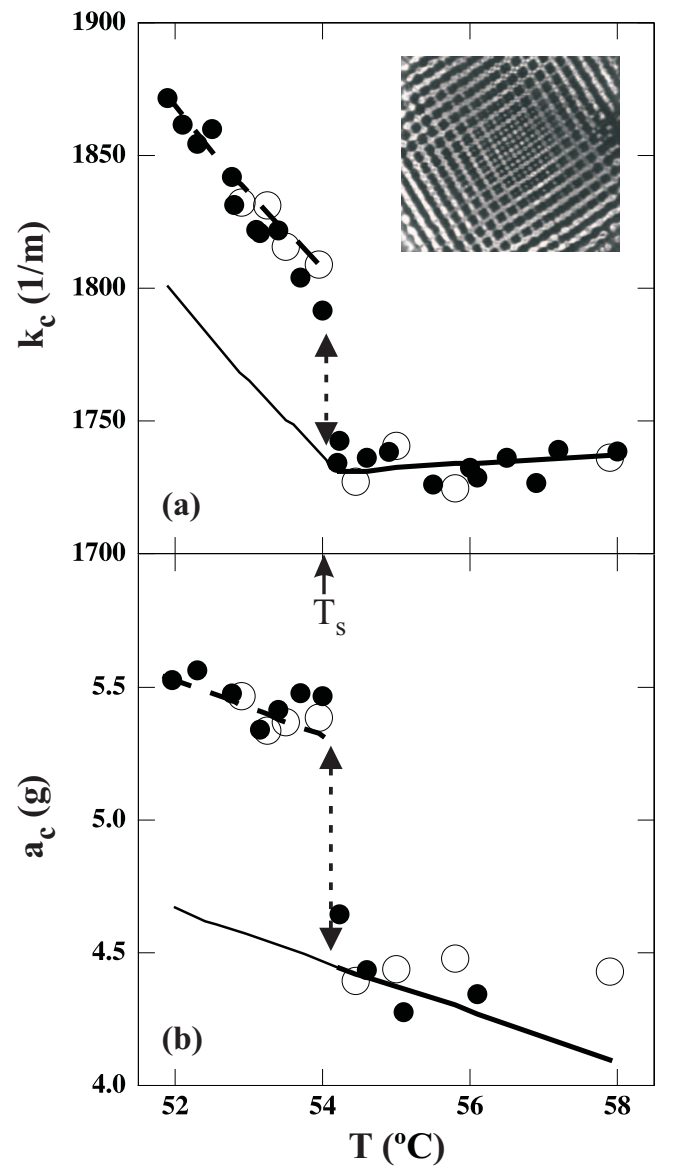

FIG. 1: Faraday instability parameters versus temperature for a fixed driving frequency $f=150 \mathrm{~Hz}$. Open (closed) symbols represent data measured on cooling (heating). (a) Critical wavenumber, $k_{\mathrm{c}}$, (b) Critical acceleration, $a_{\mathrm{c}}$. Inset to (a): Standing wave pattern at the surface at $T=54^{\circ} \mathrm{C}$. Solid and dashed lines represent the KM-model calculations assuming free-slip and no-slip boundary conditions, resp. Arrows mark the discontinuities in the Faraday parameters.

discontinuities in both quantities occur right at the onset temperature $T_{s}=54{ }^{\circ} \mathrm{C}$ reported for $\mathrm{SF}$ in $\mathrm{C}_{24} \mathrm{H}_{50}$, it is reasonable to assign these macroscopic effects to the onset of SF, a conclusion supported by the analysis below.

The measured fixed- $T f$-dependence of $k_{\mathrm{c}}$ and $a_{\mathrm{c}}$, shown in Fig. 2 is monotonically increasing, as is typical for viscous Newtonian liquids [17], both above and below $T_{s}$. Both quantities are larger for the SF phase than for the liquid surface phase over the full $f$-range investigated, in agreement with the fixed- $f, T$-dependent measurements shown in Fig. [1]

To account for these observations, we turn to surface hydrodynamics. The validity of the dispersion relation of surface waves on inviscid liquids for accurately predicting the Faraday instability dispersion relation $\left(k_{\mathrm{c}}\right.$ vs. $\left.f\right)$ of the SWP has been experimentally demonstrated [18] for liquids of viscosities $\eta \gtrsim 100 \eta_{\text {water }}$. Thus, it should also hold for liquid $\mathrm{C}_{24} \mathrm{H}_{50}$, where $\eta_{\mathrm{C}_{24} \mathrm{H}_{50}} \approx 4 \eta_{\text {water }}$. For

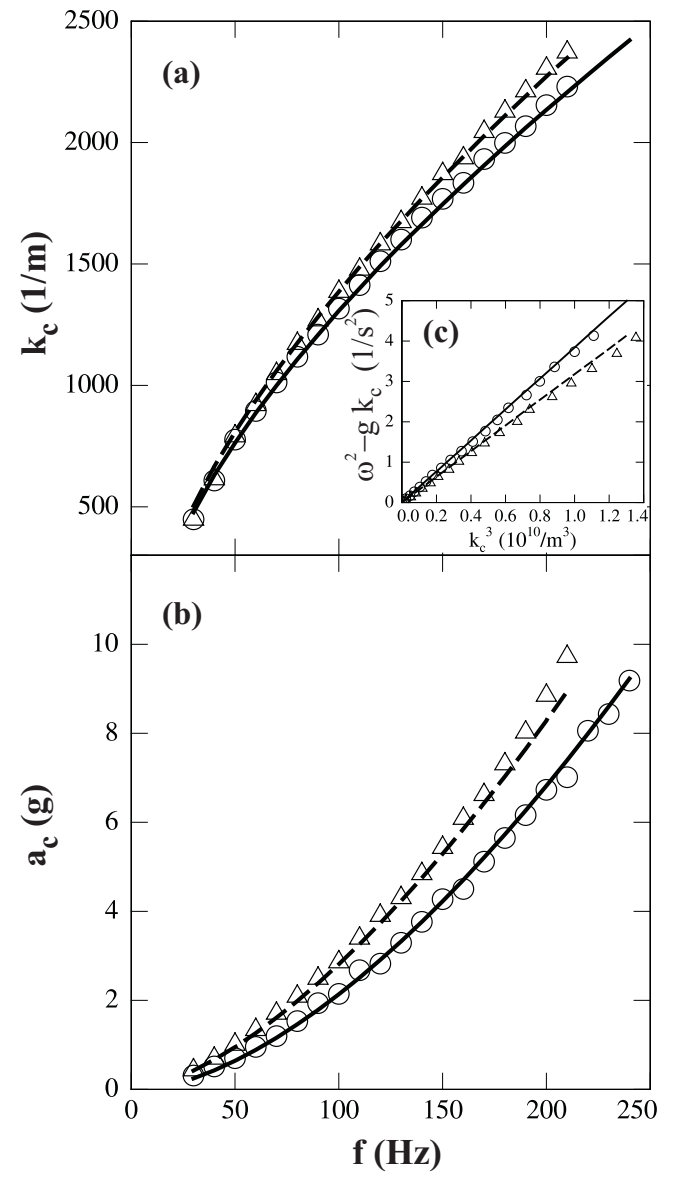

FIG. 2: Faraday instability parameters measured at $52{ }^{\circ} \mathrm{C}$ (triangles; surface-frozen state) and $58{ }^{\circ} \mathrm{C}$ (circles; nonsurface-frozen state) vs. the driving frequency, $f$ : (a) Critical wavelength, $k_{\mathrm{c}}$, (b) Critical acceleration, $a_{\mathrm{c}}$. (c) Dispersion relation plot, discussed in the text. The solid and dashed lines are calculated from the KM-model assuming free-slip and noslip boundary conditions, respectively.

a liquid layer of depth $h$ and infinite horizontal extent, this dispersion relation is $\omega^{2}=\left(g k_{\mathrm{c}}+\gamma / \rho k_{\mathrm{c}}^{3}\right) \tanh \left(k_{\mathrm{c}} h\right)$, where $g=9.81 \mathrm{~ms}^{-2}, \gamma, \rho$, and $\omega$ are the acceleration of gravity, surface tension, density, and angular frequency of the wave, respectively 19. The observed SWP in all our experiments exhibit $\omega=2 \pi f / 2$, conforming to the common subharmonic response of the Faraday instability [17. Since $\tanh \left(k_{\mathrm{c}} h\right) \approx 1$ in all our experiments, a simple linear dependence of $\omega^{2}-g k_{\mathrm{c}}$ on $k_{\mathrm{c}}^{3}$ is expected, the slope of which is $\gamma / \rho$. This relation is indeed found both above and below $T_{s}$ (Fig. 2(c)), indicating that in both the frozen and the non-frozen surface phases the simple dispersion relation is obeyed. The larger slope found at $T=58{ }^{\circ} \mathrm{C}$ as compared to that at $T=52{ }^{\circ} \mathrm{C}$ indicates that $\gamma\left(T=58{ }^{\circ} \mathrm{C}\right)>\gamma\left(T=52{ }^{\circ} \mathrm{C}\right)$, since $\rho$ does not change upon SF. This is consistent with the standard static surface tension curve $\gamma_{\mathbf{s}}(T)$, measured using the Wilhelmy plate method, and shown as a solid line in Fig. 3. The onset of SF at $T_{s}=54{ }^{\circ} \mathrm{C}$ is clearly manifested 
in this curve by the abrupt change in the slope from a negative to a positive value, due to the drop in the surface entropy upon SF [5]. Comparing this curve with the dynamic surface tension $\gamma_{\mathrm{d}}(T)$, extracted from our measurements using the dispersion relation above (Fig. [3 triangles), reveals a reasonable agreement for the nonfrozen surface phase $\left(T>T_{s}\right)$. In the SF phase $\left(T<T_{s}\right)$, however, a $\sim 3 \mathrm{mN} / \mathrm{m}$ shift of $\gamma_{\mathrm{d}}(T)$ below $\gamma_{\mathrm{s}}(T)$ is observed, although the linear $T$-dependence and the slope remain the same.

To account for this downshift, we recall that the presence of a surfactant monolayer on a liquid surface has been demonstrated to change the dispersion relation of surface waves and to increase the damping 20]. We have therefore employed a model recently introduced by Kumar and Matar for the Faraday instability in a liquid covered by an insoluble surfactant layer (KM-model) 21. Without an overlayer, the shear stress at the liquid surface vanishes, yielding a free-slip boundary condition for liquid flow. For the surfactant- or SF-layercovered surface, the shear stress is finite, and in the limit of large Marangoni number (ratio of surface-tensiongradient forces to viscous forces) yields a pinned, no-slip boundary condition. We use the KM-model with the former boundary condition for $T>T_{\mathrm{s}}$, and with the latter boundary condition for $T<T_{\mathrm{s}}$. Given the crystalline, solid structure of the surface-frozen monolayer, such an assumption appears justified. Using the measured static surface tension $\gamma_{\mathbf{s}}(T)$, the dynamic viscosity $\eta(T)$ [22], and the aforementioned change in the boundary condition at $T_{\mathrm{s}}$, the KM-model yields for fixed $f=150 \mathrm{~Hz}$ the lines shown in Fig. 1 (a) and (b). The good agreement with the measured values is evident. In particular, the change of the hydrodynamic boundary condition at $T_{\mathrm{s}}$ results not only in a jump of $\sim 20 \%$ in $a_{\mathrm{c}}$, but also in a similar jump of $\sim 3 \%$ in $k_{\mathrm{c}}$, both in good agreement with the experimental observation. Moreover, the frequency dependent calculations, shown as lines in Fig. 2] demonstrate that the KM-model accounts accurately also for the observed frequency dependence of $k_{\mathrm{c}}$ and $a_{\mathrm{c}}$ over the full dynamic range investigated, and for the abrupt increase in these quantities upon SF.

The increase in $a_{\mathrm{c}}$ upon SF reflects an increase in the damping of the surface excitations, assignable, in turn, to the large velocity gradient near the surface imposed by the pinning of the flow at the surface. The increased damping leads to a viscous detuning of the inviscid dispersion relation, and thus to a jump in the spatial periodicity of the waves. It is interesting to note that the change in the boundary condition is equivalent to an infinite surface elasticity: the surface in our model can still deform in the vertical direction, but the deformations are such that the surface neither contracts nor expands.

One of the seminal papers on SF in n-alkanes reports light-scattering measurements on thermally-excited capillary waves above and below $T_{\mathrm{s}}[23]$. The surface elon-

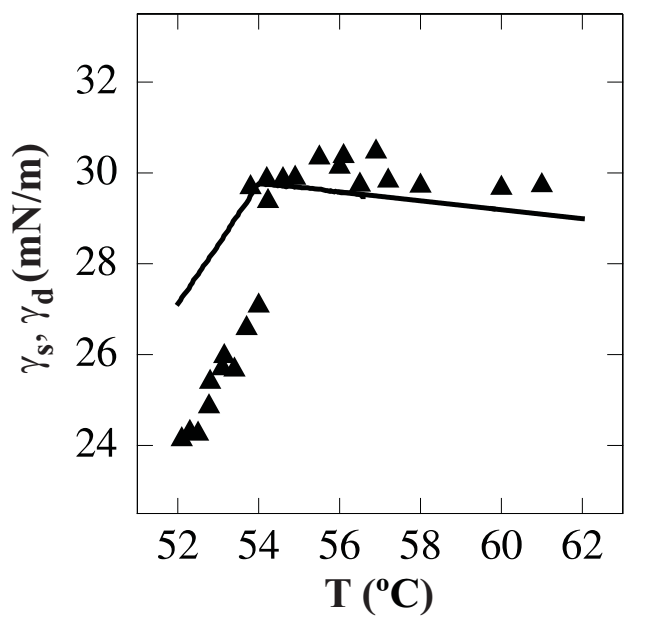

FIG. 3: Static surface tension $\gamma_{\mathrm{s}}$ measured by the Wilhelmy plate method (line) and dynamic surface tension $\gamma_{\mathrm{d}}$ (solid triangles) as determined from the surface waves' dispersion relation for a fixed $f=150 \mathrm{~Hz}$.

gations due to capillary waves are microscopic, typically of the order of nanometers 24]. However, they are still governed by the same dispersion relation as the macroscopic, mm-scale surface waves of the Faraday instability [25]. It therefore seems puzzling that for capillary waves a decreased damping was observed upon SF, rather than the increased damping found here. We believe the different behavior arises due to the different magnitudes of the surface elongations in the two cases, and consequently the different nature of the excited motion. The Faraday instability excites macroscopic waves which produce real hydrodynamic flow in the proximity of the surface. Thus, the no-slip boundary condition in the surface-frozen phase induces an increased velocity gradient, and consequently viscous dissipation across macroscopic surface-normal distances ensues. In contrast, for the nanometer-sized amplitudes of the thermally-excited capillary waves, almost no macroscopic hydrodynamic flow occurs. The postulated conformational changes in the molecular shape at $T_{\mathrm{s}}$, from a flexible, end-distorted shape to a rigid extended one 26], may even enhance the molecular mobility, particularly along their long, surfacenormal, axis. This, in turn, should reduce the damping for these microscopic excitations. Indeed, the increase in surface-normal molecular mobility, which increases the surface entropy [27], and the molecular conformation changes, which reduce the internal energy [26], are among the strongest candidates proposed for explaining the occurrence of the SF effect.

The SF-induced changes in the boundary condition, demonstrated here to alter significantly the macroscopic flow characteristics near the surface for one particular alkane, should be of importance for all materials exhibiting surface or interfacial freezing, particularly when the flow geometry involves large surface areas. One example 
is microfluidic devices [28]. Another example is foams, where the thinning and bursting rates of the bubbles are determined by the viscous drainage flow within the liquid films constituting the bubble walls 29|. Thus, the present results explain also the hydrodynamics underlying the increased lifetime of alkane bubbles, reported as a macroscopic manifestation of SF [30]. The increase in the effective viscous drag on the drainage flow, caused by the no-slip boundary condition in the SF phase, reduces the drainage rate, and consequently the wall-thinning and bursting rates of the bubbles. This leads to the observed increase in the bubbles' lifetime upon the onset of SF.

It has recently been shown that the Faraday instability can be employed to explore rheological behavior of bulk liquids 31, especially when it is difficult to use other techniques (e.g., near a liquid-vapor critical point 32]). We demonstrated here that this method allows visualizing and examining on a macroscopic scale rheological aspects of microscopic structured surfaces, in particular the interesting case of a change of the shear-stress boundary condition at a liquid's surface from the generic, free-slip one to a no-slip one, characteristic of a wetted solid wall [33]. We hope that this study will stimulate further experiments focusing on how the surface hydrodynamics is affected by microscopic modifications of the structure of surfaces or interfaces, e.g., experiments on wetting transitions which have been proven to allow for a precise control of liquid surface microstructure as a function of temperature [34]. This method could also extend the wavelength and frequency ranges of established semi-microscopic and microscopic techniques like light scattering 25] and surface x-ray photon correlation spectroscopy [35] towards macroscopic hydrodynamic length scales. More generally, the present method could be applied to other parametric instabilities to provide deeper insight into those areas of science where such instabilities occur 15.

We thank K. Knorr and M. Lücke for helpful discussions and acknowledge support by the DFG (SFB 277).

* E-mail: p.huber@physik.uni-saarland.de

[1] F. Banhart, E. Hernandez, and M. Terrones, Phys. Rev. Lett. 90, 185502 (2003); L. Zhang et al., ibid. 85, 1484 (2000).

[2] P. Huber et al., Europhys. Lett. 65, 351 (2004).

[3] M. Urbakh et al., Nature 430, 525 (2004); J.M. Drake and J. Klafter, Physics Today 43, 46 (1990).

[4] U. Raviv, P. Laurat, and J. Klein, Nature 413, 51 (2001).

[5] J.C. Earnshaw and C.J. Hughes, Phys. Rev. A 46, R4494 (1992); X.Z. Wu et al., Phys. Rev. Lett. 70, 958 (1993); E.B. Sirota et al. ibid. 79, 531 (1997) ;X.Z. Wu et al. Science, 261,1018 (1993); B.M. Ocko et al., Phys. Rev. E 55, 3164 (1997).

[6] S. Butler and P. Harrowell, Nature 415, 1008 (2002); X.Y Liu et al., ibid. 374, 342 (1995).
[7] H. Gang et al. J. Phys. Chem. B 102, 2754 (1998); M. Deutsch et al. Europhys. Lett. 30, 283 (1995); O. Gang et al., ibid. 49, 761 (2000).

[8] Q. Lei and C.D. Bain, Phys. Rev. Lett. 92, 176103 (2004).

[9] C. Merkl, T. Pfohl, and H. Riegler, Phys. Rev. Lett. 79 4625 (1997); U. Volkmann et. al. J. Chem. Phys. 116, 2107 (2002).

[10] K.S. Gautam and A. Dhinojwala, Phys. Rev. Lett. 88, 145501 (2002); K.S. Gautam et al., ibid. 90, 215501 (2003).

[11] A. Turchanin, D. Nattland, and W. Freyland, Chem. Phys. Lett. 337, 5 (2001).

[12] J. Als-Nielsen, F. Christensen, and P.S. Pershan, Phys. Rev. Lett. 48, 1107 (1982); B.M. Ocko et al., ibid. 57, 94 (1986) ; X.F. Han et al., ibid. 91, 045501 (2003).

[13] P. Lang, J. Phys.: Condens. Matter 16, R699 (2004).

[14] M. Faraday, Phil. Trans. R. Soc. London 52, 319 (1831).

[15] J. R. de Bruyn et al., Phys. Rev. Lett. 81, 1421 (1998); P. K. Shukla, ibid. 84, 5328 (2000); C. J. McKinstrie et al., Optics Express 11, 2619 (2003); V. Petrov, Qi Ouyang, and Harry L. Swinney, Nature 388, 655 (1997); G. M. Genkin, Phys. Rev. A 63, 025602 (2001).

[16] C. Wagner, H.W. Müller, and K. Knorr, Phys. Rev. E 68, 066204 (2003).

[17] K. Kumar and L. S. Tuckerman, J. Fluid Mech. 279, 49 (1994).

[18] T.B. Benjamin and J.C. Scott, J. Fluid Mech. 92, 241 (1979); W.S. Edwards and S. Fauve, ibid. 278, 123 (1994).

[19] T.B. Benjamin and F. Ursell, Proc. R. Soc. London A 225, 505 (1954).

[20] V.G. Levich, Physicochemical Hydrodynamics (PrenticeHall, Englewood Cliffs, NJ, 1962) ; J. Miles and D. Henderson, Ann. Rev. Fluid Mech. 22, 143 (1990); J.A. Nicolas, J.M. Vega, and J. Fluid Mech. 410, 367 (2000).

[21] S. Kumar and O.K. Matar, Phys. Fluids 16, 39 (2004).

[22] $\eta$ increases slightly from $4.5 \mathrm{mPa}$ s to $5 \mathrm{mPa}$ s upon cooling from $65^{\circ} \mathrm{C}$ to $52^{\circ} \mathrm{C}(\mathrm{M}$. Beiner, U. Halle-Wittenberg).

[23] C.J. Hughes and J. C. Earnshaw, Phys. Rev. E 47, 3485 (1993).

[24] A. Braslau et al. Phys. Rev. Lett. 54, 114 (1985); B.M. Ocko et al. ibid. 72, 242 (1994).

[25] Light Scattering by Liquid Surfaces and Complem. Techn., ed. by D. Langevin (Marcel Dekker, NY, 1992).

[26] A. J. Colussi, M.R. Hoffmann, and Y.C. Tang, Langmuir 16, 5213 (2000).

[27] A.V. Tkachenko and Y. Rabin, Phys. Rev. Lett. 76, 2527 (1996).

[28] H.A. Stone, A.D. Stroock, and A. Ajdari, Ann. Rev. Fluid Mech. 36, 381 (2004).

[29] G. Debregeas, P.G. de Gennes, and F. Brochard-Wyart, Science 279, 1704 (1998).

[30] H. Gang et al. Europhys. Lett 43, 314 (1998).

[31] F. Raynal et al., Eur. Phys. J. B 9, 175 (1999).

[32] S. Fauve et al., Phys. Rev. Lett. 68, 3160 (1992).

[33] E. Lauga, M.P. Brenner, and H.A. Stone, cond-mat/0501557

[34] H. Tostmann et al. Phys. Rev. Lett. 84, 4385 (2000); P. Huber et al., ibid. 89, 035502 (2002); D. Bonn and D. Ross, Rep. Prog. Phys. 64, 1085 (2001).

[35] T. Seydel et al., Phys. Rev. B 63, 073409 (2001); A. Madsen et al., Phys. Rev. Lett. 90, 085701 (2003); C. Gutt et al., Phys. Rev. Lett. 91, 076104 (2003). 